\title{
Short communication: Source tracking Bacillus cereus in an extended- shelf-life milk processing plant using partial sequencing of rpoB and multilocus sequence typing
}

\author{
Desmond T. Mugadza, Rodney Owusu-Darko, and Elna M. Buys* \\ Department of Consumer and Food Sciences, University of Pretoria, Private Bag X20, Hatfield, Pretoria 0028, South Africa
}

\section{ABSTRACT}

We used rpoB partial sequencing and multilocus sequence typing (MLST) to characterize 7 Bacillus cereus strains obtained at the following points: ESL milk during shelf life, pasteurized milk, raw milk, and filler nozzles after cleaning in place. The objective of the study was to determine relatedness among $B$. cereus isolates from several sampling points along an ESL processing plant with the aim of source tracking. The study revealed that isolates from filler nozzles shared $100 \%$ similarity with isolates from ESL milk and raw milk using rроB sequencing. It also revealed that isolates from pasteurized milk shared $100 \%$ similarities with isolates from filler nozzles and ESL milk using MLST. We suggest 3 routes of $B$. cereus contamination in ESL milk. We showed that B. cereus contamination of ESL milk might be through raw milk and biofilms from filler nozzles. In addition, $r p o B$ partial sequencing and MLST can be used as tools for source tracking in ESL milk processing.

Key words: extended shelf life milk, Bacillus cereus, source tracking, housekeeping gene

\section{Short Communication}

Despite advancement in preservation and processing technologies, Bacillus cereus remains a shelf-life and consumer safety challenge for the dairy industry (Ranieri et al., 2012; Aouadhi et al., 2014; Mugadza and Buys, 2017a). Bacillus cereus is a ubiquitous sporeforming bacterium (Ranieri et al., 2012; Aouadhi et al., 2014) that is reported to produce various toxins responsible for diarrheal and emetic food poisoning (Hansen and Hendriksen, 2001; Bartoszewicz et al.,

Received March 12, 2018.

Accepted August 22, 2018.

*Corresponding author: elna.buys@up.ac.za
2008; Stenfors Arnesen et al., 2008). Studies conducted at an extended shelf-life (ESL) milk processing plant revealed the presence of psychrotrophic $B$. cereus in milk during processing as well as during storage and in filler nozzles after cleaning in place (CIP; Khoza, 2016; Mugadza and Buys, 2017a). A follow-up study on these $B$. cereus strains then showed close relatedness between these strains using repetitive element palindromic (rep) and 16S partial sequencing (Mugadza and Buys, 2017b).

Bacillus cereus endospores can germinate at refrigeration temperatures. In addition, some strains can grow as well as produce toxins under these refrigeration conditions (Larsen and Jørgensen, 1997; Stenfors and Granum, 2001; Thorsen et al., 2006). Bacillus cereus has also been reported to be able to attach to stainless steel (Eneroth et al., 1998, 2001; Khoza, 2016), the main material used in manufacturing processing equipment. In addition, other studies have isolated $B$. cereus on processing equipment, suggesting the potential to form biofilms, which can later be responsible for postprocessing contamination of processed milk (Eneroth et al., 1998, 2001).

Although raw milk has been implicated as a source of $B$. cereus contamination in pasteurized milk (Huck et al., 2007a,b), postprocessing contamination by processing plant equipment has also been described (te Giffel et al., 1997; Svensson et al., 2000; Huck et al., 2007a,b). Identification of points of entry for these bacteria may allow the development of effective strategies for reducing or eliminating their presence in milk production systems (Huck et al., 2008).

Despite several methods being suggested for source tracking (Fu and $\mathrm{Li}, 2014$ ), multilocus sequence typing (MLST) has been described as a source tracking tool that determines exact nucleotide differences for conserved loci (Cardazzo et al., 2008). Multilocus sequence typing studies have previously been used to examine the phylogeny of the $B$. cereus complex (Helgason et al., 2000, 2004; Barker et al., 2005), identifying 3 distinct lineages that largely correspond to the species 
distribution (Cardazzo et al., 2008). In addition to MLST, partial sequencing of protein coding genes such as rpoB have also been successfully used to discriminate closely related species that are difficult to distinguish with other methods that are based on the 16S rRNA (Adékambi et al., 2009; Jiménez et al., 2013).

The objective of this research was to source track $B$. cereus in an ESL milk processing plant using partial sequencing of rpoB and MLST with the aim of determining the route of $B$. cereus contamination in ESL milk.

Seven $B$. cereus isolates obtained from various sampling points in an ESL milk processing plant: ESL milk during shelf life (BC7, BC8), pasteurized milk (BC23, $\mathrm{BC} 26)$, raw milk (BC24), as well as filler nozzles after CIP (BC5, BC29) and described in previous studies (Khoza, 2016; Mugadza and Buys, 2017a), were selected for sequencing. The isolates were selected based on previously described (GTG) ${ }_{5}$ fingerprints (Mugadza and Buys, 2017b), ensuring representation of each sampling point. Bacterial cultures were grown on nutrient agar at $30^{\circ} \mathrm{C}$ for period of 16 to $24 \mathrm{~h}$. The DNA was extracted from the $B$. cereus isolates using the ZR Fungal/Bacterial DNA MiniPrep (Zymo Research, Irvine, $\mathrm{CA}$ ) according to the manufacturer's instructions for use in the PCR reactions.

Protein coding gene rpoB together with 5 housekeeping genes, glycerol uptake facilitator protein $(g l p F)$, guanylate kinase, putative $(g m k)$, dihydroxy-acid dehydratase $(i l v D)$, phosphate acetyltransferase (pta), and phosphoribosylaminoimidazolecarboxamide (pur), distributed around the chromosome of $B$. cereus, were chosen for partial sequencing and MLST, respectively, and amplified using a Bio-Rad T100 Thermal Cycler (Singapore, Singapore). Details of primers have been previously described (Miyoshi-Akiyama et al., 2013; www.mlst.net). The reaction mixture consisted of $8 \mu \mathrm{L}$ of distilled PCR grade water, $1 \mu \mathrm{L}$ each of the forward and reverse primer, $10 \mu \mathrm{L}$ of PCR mix (containing deoxynucleotide triphosphates, buffer, $\mathrm{MgCl}_{2}$, and Taq polymerase), and $1 \mu \mathrm{L}$ of genomic DNA. The PCR protocol was as follows: $95^{\circ} \mathrm{C}$ for $5 \mathrm{~min}, 95^{\circ} \mathrm{C}$ for $30 \mathrm{~s}$, $50^{\circ} \mathrm{C}$ for $30 \mathrm{~s}, 45$ cycles, $72^{\circ} \mathrm{C}$ for $30 \mathrm{~s}, 72^{\circ} \mathrm{C}$ for $10 \mathrm{~min}$, $4^{\circ} \mathrm{C}$ hold. The PCR product was cleaned using USB ExoSAP-IT PCR Product Cleanup (Singapore, Singapore) according to the manufacturer's instructions. Gel electrophoresis was conducted using a $1 \%$ agarose gel [with $5 \mu \mathrm{L}$ of EZ-Vision In-Gel solution (Solon, OH) for every $50 \mathrm{~mL}$ of melted agarose]. The PCR product was mixed with GelRed Prestain loading buffer with orange tracking dye (Biotium Inc., Fremont, CA; https: //biotium.com/wp-content/uploads/2013/07/PI-41009 -41010.pdf). The gel was run for $30 \mathrm{~min}$ at $100 \mathrm{~V}$. The DNA sequencing was done using Big Dye V3.1
(Applied Biosystems, Foster City, CA; http://www3 .appliedbiosystems.com/cms/groups/mcb_marketing/ documents/generaldocuments/cms_081527.pdf) as per the manufacturer's instructions on the ABI $3500 \mathrm{XL}$ with POP-7 and a 50-cm array (Thermo Fisher Scientific, Waltham, MA; https://www.thermofisher.com/ order/catalog/product/A30469).

Sequence analysis was performed at Inqaba Biotechnologies (Pretoria, South Africa) and the chromatograms from the ABI 3100 sequences were exported and visually examined, and gene sequences were analyzed using FinchTV version 1.4.0 (Geospiza Inc., Seattle, $\mathrm{WA}$ ). The $r p o B$ gene sequences were aligned using BioEdit Sequence Alignment Editor version 7.2.1 (Ibis Therapeutics, Carlsbad, CA). Multiple sequence alignment was performed using Clustal Omega [EMBL-EBI, Hinxton (Conway Institute, UCD, Dublin, Ireland)]. The evolutionary history was inferred by using the maximum likelihood method based on the Jukes-Cantor model (Jukes and Cantor, 1969). The tree with the highest log-likelihood (-955.9639) was selected. Initial tree(s) for the heuristic search were obtained automatically by applying neighbor-join and BioNJ algorithms to a matrix of pairwise distances estimated using the maximum composite likelihood approach, and then selecting the topology with superior log-likelihood value. The proportion of sites where at least 1 unambiguous base is present in at least 1 sequence for each descendent clade is shown next to each internal node in the tree. The analysis involved 8 nucleotide sequences. All positions containing gaps and missing data were eliminated. Evolutionary analyses were conducted in MEGA7 (Kumar et al., 2016).

The sequences of the 5 housekeeping genes $(\mathrm{gmk}$, ilv, pur, pyc, tpi) were edited to allele lengths (between 348 and $504 \mathrm{bp}$ ). The genes sequences were then assigned allele numbers based on the already described alleles of B. cereus MLST database (http://www.pubmlst.org/ cereus). Isolates were assigned sequence type based on the combination of 5 alleles. The 5 gene fragments of each of the 7 isolates were concatenated and downloaded from the MLST website. An eighth isolate from the database, B. cereus 2053, was used to provide an outgroup sequence. The evolutionary history was inferred by using the maximum likelihood method based on the Jukes-Cantor model (Jukes and Cantor, 1969). The tree with the highest log-likelihood $(-940.3564)$ was selected. Initial tree(s) for the heuristic search were obtained automatically by applying neighbor-join and BioNJ algorithms to a matrix of pairwise distances estimated using the maximum composite likelihood approach, and then selecting the topology with superior log-likelihood value. The analysis involved 8 nucleotide 


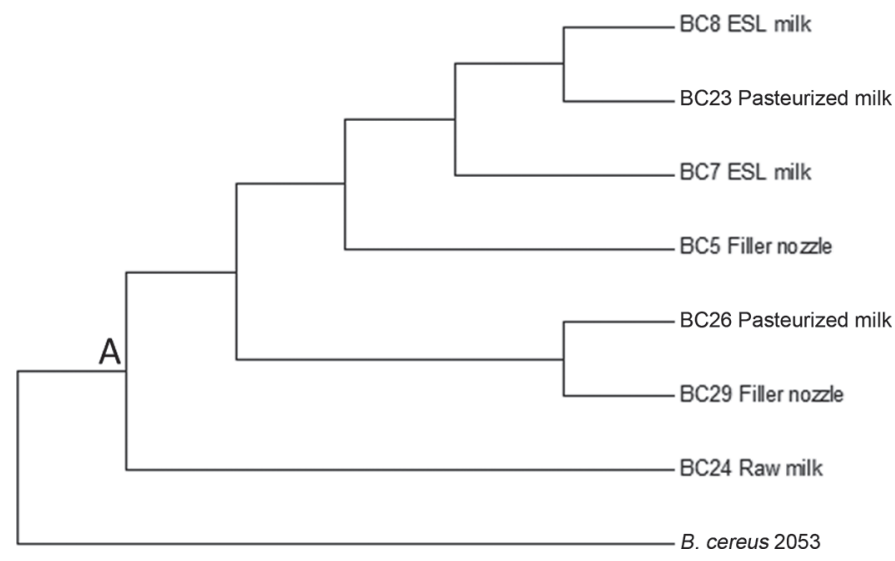

Figure 1. Multilocus sequence typing (MLST) profiles using 5 housekeeping alleles showing the relationship among Bacillus cereus strains $(\mathrm{BC})$ isolated from raw milk, pasteurized milk, extended shelflife (ESL) milk processing, and during shelf life.

sequences. All positions containing gaps and missing data were eliminated. Evolutionary analyses were conducted in MEGA7 (Kumar et al., 2016).

In this present study, B. cereus contamination routes in an ESL milk processing plant were determined using partial sequencing of the protein coding gene rpo $B$ and MSLT. The MLST showed close similarities between isolates from ESL milk (2), pasteurized milk (1), and filler nozzles (1). Two of these isolates (ESL milk and pasteurized milk) had $100 \%$ similarity showing they belonged to the same strain. Two other isolates from pasteurized milk and filler nozzles also showed $100 \%$ similarity (Figure 1).

These results show that $B$. cereus in ESL milk originated from raw milk among other sources. This is similar to previous studies that reported raw milk as the major source of $B$. cereus contamination in milk products (Huck et al., 2007a,b). The B. cereus strain from pasteurized milk was closely related to strains from ESL milk. This shows that these strains might be surviving throughout the ESL milk process until they get into the final product. Bacillus cereus strains have successfully used endospores as a strategy to survive processing hurdles as compared with their vegetative forms (Lin et al., 2017).

The MLST also revealed relatedness between $B$. cereus isolates from filler nozzles and pasteurized milk, suggesting possible postprocessing contamination by processing equipment. This is consistent with previous studies that reported the contribution of processing equipment to milk contamination (Svensson et al., 2000; Huck et al., 2007a,b). These results were also consistent with previous studies on $B$. cereus in pasteurized milk processing and farm environment that provided evidence for additional contamination of pasteurized milk in production lines (Christiansson et al., 1999; Svensson et al., 2000). In addition to endospore formation, some $B$. cereus strains also use biofilm formation together with resistance to acids and alkali as strategies of survival during CIP (Lin et al., 2017). The presence of $B$. cereus isolates from raw milk that did not cluster closely with other isolates (Figure 1) may possibly show that contamination of ESL milk is from a diverse range of sources including raw milk and ESL milk filler nozzles. This is in line with previous studies which concluded that ESL milk contamination is not exclusively from one source but rather several them including raw milk and processing equipment among others (Faille et al., 2001; Janštová et al., 2006; Miller et al., 2015). The MLST results indicate that the ancestral lineage of all the isolates was point A (Figure 1), excluding BC24. This shows that the various contaminations along the production line originated from some isolates from the raw milk. Slight genetic differences may have occurred in $B$. cereus isolates from the raw milk and those in other parts of the production line because of microevolution taking place in biofilms. This is in line with findings from previous studies that confirmed adaptation as well as horizontal gene transfer and deletion of some determinants among $B$. cereus strains (Bartoszewicz et al., 2008; Guinebretière et al., 2008; Aminov, 2011; Böhm et al., 2015).

The $r р о B$ partial sequencing showed that 3 pairs of isolates with 100\% similarity existed (Figure 2). In Figure 2, the first pair consisted of isolates from pasteurized milk, the second pair consisted of isolates from raw milk and filler nozzles, whereas the third pair had isolates from filler nozzles and ESL milk. Partial sequencing of rрoB further confirmed that $B$. cereus raw milk and pasteurized milk contributed to ESL milk contamination. This route of transmission from raw milk to pasteurized products is consistent with previous studies tracking spore-forming microbial contaminants from raw milk to finished fluid milk products (Huck et al., 2007a, 2008). Although rpoB has high discriminatory power in species and strain identification, it is important to highlight that supplementary methods might be necessary for those species that cannot be delineated by sequence comparison of a single gene (Spanu et al., 2011). Using both rpoB partial sequencing and MLST, the following 3 possible routes of $B$. cereus contamination in the ESL milk processing plant are suggested as follows: (1) B. cereus from raw milk survives the process and contaminate the final product without attaching filler nozzles, (2) B. cereus from raw milk survives the process, attaches to the filler nozzles and later contaminate the product, and (3) B. cereus 


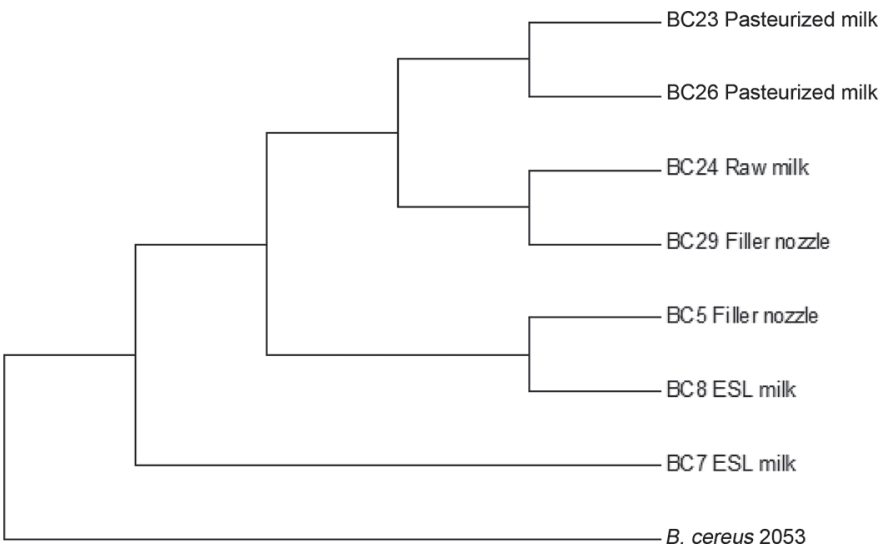

Figure 2. Neighbor-joining rpo $B$ dendrogram representing the phylogenetic relationships of Bacillus cereus strains (BC) isolated from raw milk, pasteurized milk, extended shelf-life (ESL) milk processing, and during shelf life.

from biofilms on filler nozzles contaminate the product during packaging.

The study established 3 possible routes of $B$. cereus contamination in ESL milk processing. Bacillus cereus contamination of ESL milk is through raw milk and biofilms associated with filler nozzles after CIP. However, further work will be needed using more isolates to get a more robust picture about these possible routes of $B$. cereus contamination in ESL milk. In addition, the study has also shown that rpoB partial sequencing and MLST can be used as a tool for source tracking in ESL milk processing.

\section{REFERENCES}

Adékambi, T., M. Drancourt, and D. Raoult. 2009. The rpoB gene as a tool for clinical microbiologists. Trends Microbiol. 17:37-45.

Aminov, R. I. 2011. Horizontal gene exchange in environmental microbiota. Front. Microbiol. 2:158.

Aouadhi, C., A. Maaroufi, and S. Mejri. 2014. Incidence and characterisation of aerobic spore-forming bacteria originating from dairy milk in Tunisia. Int. J. Dairy Technol. 67:95-102.

Barker, M., B. Thakker, and F. G. Priest. 2005. Multilocus sequence typing reveals that Bacillus cereus strains isolated from clinical infections have distinct phylogenetic origins. FEMS Microbiol. Lett. 245:179-184.

Bartoszewicz, M., B. M. Hansen, and I. Swiecicka. 2008. The members of the Bacillus cereus group are commonly present contaminants of fresh and heat-treated milk. Food Microbiol. 25:588-596.

Böhm, M. E., C. Huptas, V. M. Krey, and S. Scherer. 2015. Massive horizontal gene transfer, strictly vertical inheritance and ancient duplications differentially shape the evolution of Bacillus cereus enterotoxin operons hbl, cytK and nhe. BMC Evol. Biol. 15:246.

Cardazzo, B., E. Negrisolo, L. Carraro, L. Alberghini, T. Patarnello, and V. Giaccone. 2008. Multiple-locus sequence typing and analysis of toxin genes in Bacillus cereus food-borne isolates. Appl. Environ. Microbiol. 74:850-860.

Christiansson, A., J. Bertilsson, and B. Svensson. 1999. Bacillus cereus spores in raw milk: Factors affecting the contamination of milk during the grazing period. J. Dairy Sci. 82:305-314.
Eneroth, A., A. Christiansson, J. Brendehaug, and G. Molin. 1998 Critical contamination sites in the production line of pasteurised milk, with reference to the psychrotrophic spoilage flora. Int. Dairy J. 8:829-834.

Eneroth, A., B. Svensson, G. Molin, and A. Christiansson. 2001. Contamination of pasteurized milk by Bacillus cereus in the filling machine. J. Dairy Res. 68:189-196.

Faille, C., F. Fontaine, and T. Bénézech. 2001. Potential occurrence of adhering living Bacillus spores in milk product processing lines. J. Appl. Microbiol. 90:892-900.

Fu, L. L., and J. R. Li. 2014. Microbial source tracking: A tool for identifying sources of microbial contamination in the food chain. Crit. Rev. Food Sci. Nutr. 54:699-707.

Guinebretière, M. H., F. L. Thompson, A. Sorokin, P. Normand, P. Dawyndt, M. Ehling-Schulz, B. Svensson, V. Sanchis, C. NguyenThe, M. Heyndrickx, and P. De Vos. 2008. Ecological diversification in the Bacillus cereus group. Environ. Microbiol. 10:851-865.

Hansen, B. M., and N. B. Hendriksen. 2001. Detection of enterotoxic Bacillus cereus and Bacillus thuringiensis strains by PCR analysis. Appl. Environ. Microbiol. 67:185-189.

Helgason, E., H. A. Caugant, I. Olsen, and A. B. Kolst $\varnothing$. 2000. Genetic structure of population of Bacillus cereus and Bacillus thuringiensis isolates associated with periodontitis and other human infections. J. Clin. Microbiol. 38:1615-1622.

Helgason, E., N. J. Tourasse, R. Meisal, D. A. Caugant, and A.-B. Kolstø. 2004. Multilocus sequence typing for bacteria of the Bacillus cereus group. Appl. Environ. Microbiol. 70:191-201.

Huck, J. R., B. H. Hammond, S. C. Murphy, N. H. Woodcock, and K. J. Boor. 2007a. Tracking spore-forming bacterial contaminants in fluid milk processing systems. J. Dairy Sci. 90:4872-4883.

Huck, J. R., M. Sonnen, and K. J. Boor. 2008. Tracking heat-resistant, cold-thriving fluid milk spoilage bacteria from farm to packaged product. J. Dairy Sci. 91:1218-1228.

Huck, J. R., N. H. Woodcock, R. D. Ralyea, and K. J. Boor. 2007b. Molecular subtyping and characterization of psychrotolerant endospore-forming bacteria in two New York State fluid milk processing systems. J. Food Prot. 70:2354-2364.

Janštová, B., M. Draâková, and L. Vorlová. 2006. Effect of Bacillus cereus enzymes on the milk quality following ultra high temperature processing. Acta Vet. Scand. 75:601-609.

Jiménez, G., M. Urdiain, A. Cifuentes, A. López-López, A. R. Blanch, J. Tamames, P. Kämpfer, A. B. Kolstø, D. Ramón, J. F. Martínez, and F. M. Codoner. 2013. Description of Bacillus toyonensis sp. nov., a novel species of the Bacillus cereus group, and pairwise genome comparisons of the species of the group by means of ANI calculations. Syst. Appl. Microbiol. 36:383-391.

Jukes, T. H., and C. R. Cantor. 1969. Evolution of protein molecules. Pages 21-132 in Mammalian Protein Metabolism. H. N. Munro, ed. Academic Press, New York, NY.

Khoza, S. 2016. Effect of extended shelf life milk processing on the bacterial composition associated with the nozzles of filling machines. MSc dissertation, Department of Food Science, University of Pretoria, Pretoria, South Africa.

Kumar, S., G. Stecher, and K. Tamura. 2016. MEGA7: Molecular Evolutionary Genetics Analysis version 7.0 for bigger datasets. Mol. Biol. Evol. 33:1870-1874.

Larsen, H. D., and K. Jørgensen. 1997. The occurrence of Bacillus cereus in Danish pasteurized milk. Int. J. Food Microbiol. 34:179186.

Lin, Y., F. Ren, L. Zhao, and H. Guo. 2017. Genotypes and the persistence survival phenotypes of Bacillus cereus isolated from UHT milk processing lines. Food Control 82:48-56.

Miller, R., D. J. Kent, K. J. Boor, N. H. Martin, and M. Wiedmann. 2015. Different management practices are associated with mesophilic and thermophilic spore levels in bulk tank raw milk. J. Dairy Sci. 98:4338-4351.

Miyoshi-Akiyama, T., K. Hayakawa, N. Ohmagari, M. Shimojima, and T. Kirikae. 2013. Multilocus sequence typing (MLST) for characterization of Enterobacter cloacae. PLoS One 8:e66358. 
Mugadza, D. T., and E. M. Buys. 2017a. Bacillus and Paenibacillus spp. associated with extended shelf life milk during processing and storage. Int. J. Dairy Technol. 71. https://doi.org/10.1111/1471 -0307.12474 .

Mugadza, D. T., and E. M. Buys. 2017b. Diversity of Bacillus cereus strains in extended shelf life. Int. Dairy J. 73:144-150.

Ranieri, M. L., R. A. Ivy, W. R. Mitchell, E. Call, S. N. Masiello, M. Wiedmann, and K. J. Boor. 2012. Real-time PCR detection of Paenibacillus spp. in raw milk to predict shelf life performance of pasteurized fluid milk products. Appl. Environ. Microbiol. 78:5855-5863.

Spanu, T., E. De Carolis, B. Fiori, M. Sanguinetti, T. D'Inzeo, G. Fadda, and B. Posteraro. 2011. Evaluation of matrix-assisted laser desorption ionization-time-of-flight mass spectrometry in comparison to $r$ ro $B$ gene sequencing for species identification of bloodstream infection staphylococcal isolates. Clin. Microbiol. Infect. 17:44-49.

Stenfors, L. P., and P. E. Granum. 2001. Psychrotolerant species from the Bacillus cereus group are not necessarily Bacillus weihenstephanensis. FEMS Microbiol. Lett. 197:223-228.
Stenfors Arnesen,, L. P., A. Fagerlund, and P. E. Granum. 2008. From soil to gut: Bacillus cereus and its food poisoning toxins. FEMS Microbiol. Rev. 32:579-606.

Svensson, B., A. Eneroth, J. Brendehaug, G. Molin, and A. Christiansson. 2000. Involvement of a pasteurizer in the contamination of milk by Bacillus cereus in a commercial dairy plant. J. Dairy Res. $67: 455-460$

te Giffel, M. C., R. R. Beumer, L. P. M. Langeveld, and F. M. Rombouts. 1997. The role of heat exchangers in the contamination of milk with Bacillus cereus in dairy processing plants. Int. J. Dairy Technol. 50:43-47.

Thorsen, L., B. M. Hansen, K. F. Nielsen, N. B. Hendriksen, R. K. Phipps, and B. B. Budde. 2006. Characterization of emetic Bacillus weihenstephanensis, a new cereulide-producing bacterium. Appl. Environ. Microbiol. 72:5118-5121. 